\title{
The Menace of Lead Poisoning in Pakistan
}

\author{
Rasheed MA* \\ Pharmacology and Toxicology Department, University of Veterinary and Animal \\ Sciences Lahore, Pakistan
}

*Corresponding author: Muhammad Adil Rasheed, Assistant Professor, Department of Pharmacology and Toxicology, Sheikh Abdul Qadir Jillani (Out Fall)

Road, University of Veterinary and Animal Sciences Lahore, Pakistan, Tel: 00923218390727; Email: dr_aadil@uvas.edu.pk

\section{Introduction}

Environmental pollution has been increased many times due to increase in population, industrialization and urbanization; in some places, it becomes toxic to living organisms. Among the pollutants, heavy metals pose the highest threat to biological systems as they are inorganic and non-biodegradable. Unlike essential heavy metals, mercury, lead, cadmium and arsenic are very toxic to living organisms even at low concentrations. In Pakistan, health problems have been reported due to exposure of heavy metals from contaminated soil, air, water, food and urban road dusts. Lead $(\mathrm{Pb})$ is one of the poisonous heavy metal that is the leading cause of environmental pollution especially in developing countries like Pakistan. The presence of lead in the environment is due to its nonbiodegradable nature. Continuous exposure of lead even at low level can cause serious health concerns in children as well in adults. The intensity of lead poisoning mainly depends upon its source, nature, route of exposure and frequency of exposure. Moreover, lead has versatile properties such as poor conductibility, softness and resistance to corrosion makes it suitable for use in different industries by man over 6000 years, resulting in increased hazards of lead poisoning. Exposures of lead from various sources cause a serious health concern, known as lead poisoning or plumbism. Lead is extremely poisonous metal that affects almost every organ of the body. Nervous system is the most affected organ by lead both in adults and children. According to Centre of Disease Control (CDC), in blood, $\geq 5 \mu \mathrm{g} / \mathrm{dl}$ is considered highly toxic equally for adults and children.

\section{Sources of Lead}

Older buildings and apartments having lead based paint is the leading source of lead toxicity in children. Household furniture, toys, batteries, sanitary pipes, cosmetics (Tiro, kohl, surma), canned food are the other major sources of lead toxicity in humans and have been banned by the US government since 1978. Sanitary pipes can release lead particles in drinking water as they contain lead as impurities. Furthermore, Mexican candies are linked with lead toxicity. Occupational toxicity can also occur in people, work in mining, painting, batteries manufacturing and auto mobile repairing.

\section{Exposure Routes}

Entry of lead into human body is mainly through food, water and air followed by its acute or chronic poisoning. Surface water in different regions of Pakistan found contamination of lead beyond the permissible limits according to World health organization followed by ground water. Further, lead is associated with more than 900 occupations including smelting, mining, battery manufacturing, lead based paint industries, plastic manufacturing, plumbing and pipe fitting, printing and auto repairing etc. During the $20^{\text {th }}$ century, lead pollution was at peak as tetraethyl lead was extensively used to enhance the octane number of gasoline. Around 1970's, the total production of tetraethyl lead (TEL) in world was increased up to 500,000 tonnes. Lead in gasoline was one of the major cause of environmental pollution in past. Since the use of TEL in gasoline was banned, the worldwide production of TEL was dramatically reduced 


\section{Advances in Clinical Toxicology}

approximately up to 45,000 tonnes. According to scientists, In United States, lead based gasoline is the only major cause of lead deposition (4-5 million tonnes) in the soil. Lead based paints, after the ban on production of leaded gasoline, are the main source lead exposure globally.

\section{Absorption of Lead}

Absorption of lead mainly depends upon its source, physiochemical properties of lead and condition of exposed person. Inorganic lead after absorption not metabolized but distributed to soft tissues and bones. Lead toxicity usually remains asymptomatic. It was reported that about $20-70 \%$ of ingested lead in adults enters the bloodstream in comparison to inhaled lead as absorption of inhaled lead is almost 100\%. Young children, pregnant females, essential metal deficient people are at high risk of lead toxicity due to increase rate of absorption. After 40 days, circulating lead in deposited in bones and teeth. The rate of deposition of lead in bones and teeth is greater in adults than in children. As remodeling of bones takes place in children, lead from bones move towards blood stream and manifest the toxic symptoms of lead.

\section{Pathophysiological Manifestations}

Lead has toxic effect on almost all vital organ systems of the body including cardiovascular system, nervous system, renal system, skeletal system, reproductive system, immune system etc. In short, there is almost no function of human body which is not affected by lead toxicity. Nervous system is primarily the most affected part of human body due to lead toxicity. Pb has ability to cross placental barrier in those pregnant women exposed to lead and cause the developmental abnormalities in children.

\section{Mechanism of Toxicity}

The main pathway of lead toxicity is to block calcium actions and capability to react with biological proteins. $\mathrm{Pb}$ has also ability to inhibit the enzymes activity by altering the structure such as sulfhydryl enzymes. Further, $\mathrm{Pb}$ can induce oxidative stress, one of the major mechanisms of lead toxicity. The intensity of damage due to lead depends upon the blood lead levels. There is no symptom of lead toxicity reported in both males and females below $5 \mu \mathrm{g} / \mathrm{dl}$ blood lead level. In contrast, more than $20 \mu \mathrm{g} / \mathrm{dl}$ blood lead level is responsible for kidney damage, hypertension, subclinical neurocognitive defects, spontaneous abortion, anemia, sperm abnormalities, encephalopathy and reduced birth weight.

\section{Oxidative stress}

Lead induces oxidative stress by deteriorating naturally occurring antioxidant systems of the body as lead is known to have a strong property of covalent bond formation. Antioxidant enzymes containing sulfhydryl groups are the most common targets for lead. The covalent bonds are formed between the lead moiety and the sulfhydryl groups, ultimately making them inactive. The enzymes which are inactivated by lead include aminolevolinic acid dehydratase (ALAD), glutathione reductase, glutathione S- transferase, superoxide dismutase and catalase. The reactive oxygen species (ROS) which are produced due to exposure of lead involve in the lipid peroxidation of membranes of red blood cells. As a result, denaturation of RBCs occurs due to excessive production of ROS leading towards the hemolytic or frank anemia. The enzymes involved in the synthesis of heme are adversely affected by both acute and chronic lead toxicity.

\section{Neurological Effects}

Various reports provide enough evidence about the adverse effects of lead on the central nervous system especially on brain. Cognitive and physiological changes in the brain are observed followed by lead toxicity. Children are at high risk because of almost 50\% absorption of water soluble lead ingestion. Lead exposure to children even at very low concentration is associated with decreased academic performance, learning capacity, intellectual development and onset of behavioral problems, revealed by many studies. However, chronic exposure of lead in high concentration leads to neuropsychiatric problems i.e. attention deficit and antisocial behavior. In short, lead toxicity in children has immediate and long terms consequences on the mental health. Lead has two types of toxic effects on nervous system, one is morphological and other is pharmacological. Morphological changes include the decreased synthesis of neuronal sialic acid. Sialic acid is the key molecule that interferes with synapse formation. Pharmacological effects of $\mathrm{Pb}$ accumulation include the interference in release of neurotransmitters, inhibition of $\mathrm{N}$-methyl-D-aspartate (NMDA) ion channels and substitution of calcium and zinc.

The mechanism of neurotoxicity caused by lead is not fully understood. Lead altered the cell signaling mechanism and neurotransmission followed by 
neurotoxicity possibly due to oxidative stress and impairment of antioxidants. Moreover, lead is responsible for disruption of blood brain barrier (BBB) as it can modulate the protein kinase $\mathrm{C}$ pathway. It also acts as analogue of calcium ion and concentrates in brain by crossing $\mathrm{BBB}$ and alters the functioning of glutamate, which has vital role for learning behavior inside the brain.

\section{Diagnosis}

Different strategies (Basophilic stripping, erythrocyte protoporphyrin measurement and X-Rays fluorescence technique) are useful for detection of lead poisoning. Basophilic stripping is one of the important sign of lead poisoning as dots in red blood cells are seen under microscope after examination of blood film of individual exposed to lead. Other useful technique for $\mathrm{Pb}$ toxicity detection is the measurement of erythrocyte protoporphyrin (EP) in blood samples as it tends to increase when blood lead level is high. Although, EP level is not sufficient enough to detect elevated blood lead levels below $35 \mu \mathrm{g} / \mathrm{dL}$. Due to less sensitivity and higher threshold, the use of this method for detecting lead toxicity has been decreased. Blood lead levels only provide an idea for recent lead exposure. The cumulative and total body burden of lead can be measured best by Xray fluorescence technique. Paint chips can also revealed by X-rays in gut of children.

\section{Prevention and Treatment}

Lead as such has no physiological role in the body and even smaller levels of lead can cause deleterious effects. The good aspect is however that it can be reversed by a number of techniques used nowadays the recommended treatment of acute lead poisoning is chelation therapy. Different chelating agents are useful to cure lead toxicity. Chelating agents e.g. dimercaprol and succimer have capability to bind with lead to form nontoxic complexes and hence, decrease the lead burden. Other chelating agents include $\mathrm{CaNa}_{2}$ EDTA, D-penicillamine and DMSA. Moreover, antioxidant therapy is also useful to cure the toxicity lead. Nano-encapsulation of antioxidants is a modern technique that ensures the good bioavailability and distribution of poorly absorbed compounds. 